\title{
Adult Pineal Gland Astrocytoma
}

National Cancer Institute

\section{Source}

National Cancer Institute. Adult Pineal Gland Astrocytoma. NCI Thesaurus. Code

C115327.

A pineal gland astrocytoma that occurs during adulthood. 